\title{
NEW RESULTS ON POWER-ASSOCIATIVE ALGEBRAS ${ }^{(1)}$
}

\author{
BY
}

\section{LOUIS A. KOKORIS}

1. Introduction. Many of the results concerning power-associative commutative rings and algebras carry the restriction that the characteristic be prime to $30[1 ; 2 ; 3]\left({ }^{2}\right)$. We shall study the cases where the characteristic is 3 or 5 and shall show that the results are those of the general case if we make a slight modification of the definition of power-associativity. However, our proofs require the use of the associativity of fourth, fifth, and sixth powers, while the results for characteristic prime to 30 use only the associativity of fourth powers.

It is known that there exist simple commutative power-associative algebras of degree two and characteristic $p>5$ which are not Jordan algebras $[4 ; 5]$. We shall obtain the important property of algebras of degree two and characteristic zero given in Theorem 6 . It is hoped that this result may lead to a proof of the conjecture that a simple power-associative commutative algebra of degree two and characteristic zero is necessarily a Jordan algebra.

We shall assume from the outset that the system under consideration is commutative and has characteristic not two.

2. Definitions and identities. If $x^{\alpha} x^{\beta}=x^{\alpha+\beta}$ for every $x$ of $\mathfrak{A}$ and integers $\alpha$ and $\beta$, then $(x+\lambda y)^{\alpha}(x+\lambda y)^{\beta}=(x+\lambda y)^{\alpha+\beta}$ for all $x$ and $y$ in $\mathfrak{A}$, where $\lambda$ is any integer in case $\mathfrak{A}$ is a ring, and $\lambda$ is any element of the base field if $\mathfrak{A}$ is an algebra. The result obtained by this substitution is a polynomial $\sum_{\substack{i=0 \\ \alpha+\beta}}^{a} \lambda_{i}$ $=0$ in $\lambda$. Each $A_{i}$ is called an attached polynomial of $\mathfrak{A}$ and further linearization yields other attached polynomials. We use these facts in the following definitions.

Definition 1 . A commutative ring $\mathfrak{A}$ will be said to be strictly powerassociative if $x^{\alpha} x^{\beta}=x^{\alpha+\beta}$ for every $x$ of $\mathfrak{A}$ and all integers $\alpha$ and $\beta$ and if every attached polynomial of $\mathfrak{A}$ is zero.

When $\mathfrak{A}$ is an algebra, Definition 1 is equivalent to

Defrition 2. A commutative algebra $\mathfrak{A}$ over a field $\mathfrak{F}$ is called strictly power-associative if $x^{\alpha} x^{\beta}=x^{\alpha+\beta}$ for all positive integers $\alpha$ and $\beta$, and every $x$ of $\mathfrak{A}_{\Re}$ where $\Re$ is any scalar extension of $\mathfrak{F}$.

Let us now consider the associativity of fourth powers; that is, the identity $A(x) \equiv x^{2} x^{2}-\left(x^{2} x\right) x=0$. Linearization of $A(x)$ gives the identity

$$
4(x y) x^{2}=2[(x y) x] x+\left(x^{2} y\right) x+x^{3} y
$$

Presented to the Society, December 27, 1951; received by the editors December 29, 1952.

(1) This work was carried out in part with the aid of the Office of Naval Research.

(2) Numbers in brackets refer to the bibliography at the end of the paper. 
for a ring $\mathfrak{A}$ whose characteristic is greater than 3 and for an algebra $\mathfrak{A}$ over a field $\mathfrak{F}$ whose characteristic is 3 and which contains more than three elements. We also obtain

$$
\begin{aligned}
4(y z) x^{2}+8(x y)(x z)= & 2[(y z) x] x+2[(x y) z] x+2[(x y) x] z \\
& +2[(x z) y] x+2[(x z) x] y+\left(x^{2} y\right) z+\left(x^{2} z\right) y
\end{aligned}
$$

and

$$
\begin{aligned}
4[(x y)(z w)+ & (x z)(y w)+(x w)(y z)] \\
= & x[y(z w)+z(w y)+w(y z)]+y[x(z w)+z(w x)+w(x z)] \\
& +z[x(y w)+y(w x)+w(x y)]+w[x(y z)+y(z x)+z(x y)]
\end{aligned}
$$

without any restrictions on the ring $\mathfrak{A}$.

At this point we note that when the characteristic is prime to 30 , strict power-associativity is equivalent to power-associativity. This follows from the fact that associativity of fourth powers implies the associativity of all higher powers [1], and fourth power associativity is equivalent to the multilinear identity (3).

To show that strict power-associativity is not equivalent to powerassociativity we consider the commutative free algebra $\mathfrak{A}$ of all polynomials in $x$ and $y$ over the field $\mathfrak{F}$ of three elements. Restrict $\mathfrak{A}$ by defining all products to be zero except $x, x^{2}, x^{3}, y, y^{2}, y^{3}, x y,(x y) x,(x y) y, x^{2} y, y^{2} x,\left(y^{2} x\right) y, x^{3} y$, and let $\left(y^{2} x\right) y=-x^{3} y$. Computation shows that $\mathfrak{A}$ is power-associative, but, since (1) is not satisfied, is not strictly power-associative.

The assumption of the associativity of fifth powers gives the identity

$$
\begin{aligned}
2[(x y) x] x^{2}+\left(x^{2} y\right) x^{2} & +2(x y) x^{3} \\
& =x^{4} y+\left(x^{3} y\right) x+\left[\left(x^{2} y\right) x\right] x+2\{[(x y) x] x\} x
\end{aligned}
$$

under the restrictions that applied to relation (1). Relation (4) yields

$$
\begin{aligned}
2[(x y) z+(y z) x & +(z x) y] x^{2}+2\left[2(x y) x+x^{2} y\right](x z) \\
& +2\left[2(x z) x+x^{2} z\right](x y)+2(y z) x^{3} \\
= & \left\{2[(x y) z+(y z) x+(x z) y] x+\left[2(x z) x+x^{2} z\right] y\right. \\
& \left.+\left[2(x y) x+x^{2} y\right] z\right\} x+\left\{2[(x z) x] x+\left(x^{2} z\right) x+x^{3} z\right\} y \\
& +\left\{2[(x y) x] x+\left(x^{2} y\right) x+x^{3} y\right\} z
\end{aligned}
$$

and a relation which may be summarized by

$$
\sum\left[\left(x_{1} x_{2}\right) x_{3}\right]\left(x_{4} x_{5}\right)=\sum\left\{\left[\left(x_{1} x_{2}\right) x_{3}\right] x_{4}\right\} x_{5}
$$

with two factors of each summand equal to $x$ and the remaining factors one each to $y, z$, and $w$.

It will be necessary to use an identity derived from the equality $x^{4} x^{2}=x^{5} x$. This identity is obtained with the same restrictions that apply to (1) and 
may be written as

$$
\sum\left\{\left[\left(x_{1} x_{2}\right) x_{3}\right] x_{4}\right\}\left(x_{5} x_{6}\right)=\sum\left(\left\{\left[\left(x_{1} x_{2}\right) x_{3}\right] x_{4}\right\} x_{5}\right) x_{6}
$$

with three factors of each summand equal to $x$ and the remaining factors one each to $y, z$, and $w$.

3. Conditions for the associativity of powers. Albert has shown [1] that the associativity of fourth powers implies the associativity of all higher powers in a commutative ring whose characteristic is prime to 30. Furthermore he has given examples of commutative rings of characteristic 3 and of characteristic 5 which satisfy $x^{2} x^{2}=x^{3} x$ but with not all higher powers associative. The additional conditions which must be imposed on rings of characteristic 3 or 5 are given in the following two theorems.

THEOREM 1. Let $\mathfrak{A}$ be a commutative algebra over a field $\mathfrak{F}$ whose characteristic is $3, \mathfrak{F}$ have more than three elements, and $x^{2} x^{2}=x^{3} x, x^{3} x^{2}=x^{4} x$ for every $x$ in $\mathfrak{A}$. Then $\mathfrak{A}$ is power-associative.

For proof we first observe that the hypotheses imply (2), (4), and (5). Now let $y=x^{k-1}$ and $z=x^{n-k-1}$ in (2) for $k=2,3$ and obtain, after using $x^{\lambda} x^{\mu}=x^{\lambda+\mu}$ for $\lambda+\mu<n, x^{n-4} x^{4}=x^{n-3} x^{3}=x^{n-2} x^{2}$. Next replace $y$ in (4) by $x^{n-4}$ and then have $2 x^{n-3} x^{3}=x^{n-4} x^{4}+x^{n-1} x$. Thus $x^{n-2} x^{2}=x^{n-1} x$.

The equality $x^{n-v} x^{\nu}=x^{n-1} x$ clearly holds for $\nu=1,2,3,4$. Assume it for $\nu=1,2, \cdots, k$. Then take $y=x^{k-2}, z=x^{n-(k+1)}$ in (5) so that $2 x^{n-3} x^{3}$ $=x^{n-(k+2)} x^{k-2}+x^{n-(k+1)} x^{k+1}$. We use the hypothesis of the induction and then have $x^{n-(k+1)} x^{(k+1)}=x^{n-1} x$ as desired.

The proof depends on the fact that when $\mathfrak{A}$ is a commutative algebra over a field $\mathfrak{F}$ of more than three elements, $x^{3} x^{2}=x^{4} x$ implies (4) which in turn implies (5). For rings we assume (4) and obtain the following result.

COROLlaRy. Let $\mathfrak{A}$ be a commutative ring whose characteristic is 3 and let $x^{2} x^{2}=x^{3} x$ and (4) hold. Then $\mathfrak{A}$ is power-associative.

Theorem 2. Let $\mathfrak{A}$ be a commutative ring whose characteristic is $\mathbf{5}$ and let $x^{2} x^{2}=x^{3} x$ and $x^{4} x^{2}=x^{5} x$. Then $\mathfrak{A}$ is power-associative.

Replace the variables in (3) by powers of $x$ with positive exponents $\alpha, \beta$, $\boldsymbol{\gamma}, \boldsymbol{\delta}$ and obtain

$$
\begin{aligned}
4\left[x^{n-(\alpha+\beta)} x^{\alpha+\beta}+\right. & \left.x^{n-(\alpha+\gamma)} x^{\alpha+\gamma}+x^{n-(\beta+\gamma)} x^{\beta+\gamma}\right] \\
& =3\left[x^{n-\alpha} x^{\alpha}+x^{n-\beta} x^{\beta}+x^{n-\gamma} x^{\gamma}+x^{n-(\alpha+\beta+\gamma)} x^{\alpha+\beta+\gamma}\right]
\end{aligned}
$$

whenever $x^{\lambda} x^{\mu}=x^{\lambda+\mu}$ for $\lambda+\mu<n=\alpha+\beta+\gamma+\delta$.

The values $\alpha=\beta=\gamma=1$ in (8) give

$$
2 x^{n-2} x^{2}=4 x^{n-1} x+3 x^{n-3} x^{3} .
$$

Let $n=5$ so that $2 x^{3} x^{2}=4 x^{4} x+3 x^{3} x^{2}, x^{3} x^{2}=x^{4} x$, and thus the associativity of 
fourth powers implies the associativity of fifth powers in a commutative ring whose characteristic is 5 . If we set $n=6$ in (9), $2 x^{4} x^{2}=4 x^{5} x+3 x^{3} x^{3}$, and our hypothesis on sixth powers implies the associativity of all sixth powers. We now have $x^{\lambda} x^{\mu}=x^{\lambda+\mu}$ for $\lambda+\mu<n, n \leqq 7$.

The substitution $\alpha=2, \beta=\gamma=1$ in (8) yields

$$
x^{n-4} x^{4}=x^{n-3} x^{3}+2 x^{n-2} x^{2}+3 x^{n-1} x,
$$

and the result of setting $\alpha=3, \beta=\gamma=1$ is

$$
x^{n-5} x^{5}=x^{n-4} x^{4}+4 x^{n-3} x^{3}+3 x^{n-2} x^{2}+3 x^{n-1} x .
$$

Since we have assumed the associativity of sixth powers we may take $\alpha+\beta+\gamma$ $=6$. We use the values $\alpha=\beta=\gamma=2$ and $\alpha=3, \beta=2, \gamma=1$ to obtain $x^{n-6} x^{6}$ $=4 x^{n-4} x^{4}+2 x^{n-2} x^{2}=3 x^{n-5} x^{5}+3 x^{n-4} x^{4}+2 x^{n-3} x^{3}+4 x^{n-2} x^{2}+4 x^{n-1} x$. Then use (11) and (10) to successively eliminate $x^{n-5} x^{5}$ and $x^{n-4} x^{4}$ from the last expression and have $x^{n-3} x^{3}=x^{n-1} x$. Relation (9) implies $x^{n-2} x^{2}=x^{n-1} x$.

Clearly $x^{n-\nu} x^{\nu}=x^{n}$ for $\nu=1,2,3$. Assume the equality for $\nu=1,2, \cdots, k$ and then let $\alpha=k-1, \beta=\gamma=1$ in (8). This substitution gives $3 x^{n-k} x^{k}+4 x^{n-2} x^{2}$ $=3 x^{n-(k-1)} x^{k-1}+x^{n-1} x+3 x^{n-(k+1)} x^{k+1}$. By the induction hypothesis $x^{n-(k+1)} x^{k+1}$ $=x^{n}$ and this completes the proof.

4. Decomposition relative to an idempotent. In a commutative strictly power-associative ring $\mathfrak{A}$ with an idempotent $e$ we have relation (1). Let $y=e$ in (1) to obtain a result which, when written in terms of right multiplications, is

$$
2 R_{e}^{3}-3 R_{e}^{2}+R_{e}=R_{e}\left(R_{e}-I\right)\left(2 R_{e}-I\right)=0 .
$$

As in the general case [2] the relation (12) implies the decomposition of $\mathfrak{A}$ into the supplementary sum $\mathfrak{A}=\mathfrak{A}_{e}(1)+\mathfrak{A}_{e}(1 / 2)+\mathfrak{A}_{e}(0)$. We may then obtain the following theorem giving multiplicative relations between the modules $\mathfrak{A}_{e}(\lambda)$ for rings with characteristic 3 or 5 . The statement is that of the general case [2] and, since much of the proof is the same, we shall prove only the parts for which the general proof does not hold.

Theorem 3. The modules $\mathfrak{A}_{e}(1)$ and $\mathfrak{A}_{e}(0)$ in a commutative strictly powerassociative ring $\mathfrak{A}$ are zero or orthogonal subrings of $\mathfrak{A}$. They are related to $\mathfrak{H}_{e}(1 / 2)$ by the inclusion relations

$$
\begin{aligned}
\mathfrak{A}_{e}(1 / 2) \mathfrak{A}_{e}(1 / 2) & \subseteq \mathfrak{A}_{e}(1)+\mathfrak{A}_{e}(0), \\
\mathfrak{A}_{e}(1) \mathfrak{A}_{e}(1 / 2) & \subseteq \mathfrak{A}_{e}(1 / 2)+\mathfrak{A}_{e}(0), \\
\mathfrak{A}_{e}(0) \mathfrak{A}_{e}(1 / 2) & \subseteq \mathfrak{A}_{e}(1 / 2)+\mathfrak{A}_{e}(1) .
\end{aligned}
$$

We first consider the case where $\mathfrak{A}$ has characteristic 3 . The values $z=w$ $=e, x e=\lambda x, y e=\mu y$ in (3) give a relation which may be written as

$$
(x y)\left[2 R_{e}^{2}+(2 \lambda+2 \mu-4) R_{e}+\left(2 \lambda^{2}+2 \mu^{2}+\lambda+\mu-8 \lambda \mu\right) I\right]=0 .
$$


The substitution $x=e, z e=\lambda z, y e=\mu y$ in (5) results in a relation which, after writing $x$ for $z$, is

$$
\begin{aligned}
& (x y)\left[2 R_{e}^{3}+(2 \lambda+2 \mu-2) R_{e}^{2}+\left(2 \lambda^{2}+2 \mu^{2}-\lambda-\mu-2\right) R_{c}\right. \\
& \left.+\left(2 \lambda^{3}+2 \mu^{3}+\lambda^{2}+\mu^{2}+\lambda+\mu-4 \lambda^{2} \mu-4 \lambda \mu^{2}-4 \lambda \mu\right) I\right]=0 .
\end{aligned}
$$

When $\lambda=\mu=1,(14)$ and (15) become $(x y)\left[2 R_{e}^{2}-2 I\right]=(x y)\left[2 R_{e}^{3}+2 R_{e}^{2}-4 I\right]$ $=0$. By (19), $R_{e}^{3}=R_{e}$ so $(x y)\left[R_{e}^{2}-I\right]=(x y)\left[R_{e}^{2}+R_{e}+I\right]=0$. Consequently $(x y)\left[R_{e}-I\right]=0$ and $\mathfrak{A}_{e}(1)$ is a subring of $\mathfrak{A}$. The values $\lambda=\mu=0$ in (15) yield $(x y)\left[2 R_{e}^{3}-2 R_{e}^{2}-2 R_{e}\right]=0=(x y) R_{e}^{2}$ and therefore $\mathfrak{A}_{e}(0)$ is a subring of $\mathfrak{A}$. Next let $\lambda=0, \mu=1$ in (14) and (15) to obtain $(x y)\left[2 R_{e}^{2}-2 R_{e}\right]=(x y)\left[2 R_{e}^{3}-R_{e}+4 I\right]$ $=0$. It follows that $(x y)\left[R_{e}+I\right]=0,(x y)\left[R_{e}^{2}+R_{e}\right]=0$ and, since $(x y)\left[R_{e}^{2}-R_{e}\right]$ $=0,(x y) R_{e}=0$. Thus $(x y) I=0$; that is, $\mathfrak{A}_{e}(1)$ and $\mathfrak{A}_{e}(0)$ are orthogonal.

Let $\mathfrak{A}$ have characteristic 5 . It is only necessary to show the orthogonality of $\mathfrak{A}_{e}(1)$ and $\mathfrak{A}_{e}(0)$. To this end set $w=x=e, y e=y, z e=0$ in (7) and so obtain $(x y)\left[2 R_{e}^{4}+R_{e}^{2}-R_{e}\right]=0$ where we have replaced $z$ by $x$. By (12), $2 R_{e}^{4}=R_{e}^{2}+R_{e}$ so $(x y) R_{e}^{2}=0,(x y) R_{e}=0$. If $\lambda=0, \mu=1$ in (14), (xy) $\left[2 R_{e}^{2}-2 R_{e}+3 I\right]=0$ and consequently $x y=0$ as desired.

5. On certain mappings and their properties. The purpose of the next part of our work is to show that the results of [3] on certain mappings hold for rings and algebras of characteristic 3 or 5 . The statements of the results are exactly those given for rings or algebras with characteristic prime to 30 , except that we shall be working with strictly power-associative systems. However, some of the proofs given in [3] are not valid when the characteristic is 3 or 5 , and we shall concern ourselves with furnishing proofs in these cases. Furthermore, we shall adopt the notations of [3].

Much of our work will depend on the mappings $S_{0}\left(x_{1}\right), S_{1 / 2}\left(x_{1}\right), T_{1}\left(x_{0}\right)$, and $T_{1 / 2}\left(x_{0}\right)$ defined in [3] and relations (5), (6), (7), and (8) of [3]. Of these relations the first of (5), the first of (6), and all of (8) must be shown for a ring whose characteristic is 3 . Also, (7) must be proved when the characteristic is 5 .

These relations are obtained in a straightforward manner from the identities of $\S 2$, so there is no need to give details. The substitution $x=u=u^{2}$, $y=y_{1}, z=z_{1}, w=w_{1 / 2}$ in (6), where $a_{\lambda}$ is in $\mathfrak{A}_{u}(\lambda)$, gives the following pair of relations when the characteristic is 3 .

$$
\begin{aligned}
S_{1 / 2}\left(z_{1} y_{1}\right) & =S_{1 / 2}\left(z_{1}\right) S_{1 / 2}\left(y_{1}\right)+S_{1 / 2}\left(y_{1}\right) S_{1 / 2}\left(z_{1}\right), \\
S_{0}\left(z_{1} y_{1}\right) & =2 S_{1 / 2}\left(z_{1}\right) S_{0}\left(y_{1}\right)+2 S_{1 / 2}\left(y_{1}\right) S_{0}\left(z_{1}\right) .
\end{aligned}
$$

A corresponding pair of relations is obtained by setting $x=u, y=y_{0}, z=z_{0}$, and $w=w_{1 / 2}$ in (6). These are

$$
\begin{aligned}
T_{1 / 2}\left(z_{0} y_{0}\right) & =T_{1 / 2}\left(z_{0}\right) T_{1 / 2}\left(y_{0}\right)+T_{1 / 2}\left(y_{0}\right) T_{1 / 2}\left(z_{0}\right), \\
T_{1}\left(z_{0} y_{0}\right) & =2 T_{1 / 2}\left(z_{0}\right) T_{1}\left(y_{0}\right)+2 T_{1 / 2}\left(y_{0}\right) T_{1}\left(z_{0}\right) .
\end{aligned}
$$


It is necessary to set $x=u, y=y_{1}, z=z_{0}, w=w_{1 / 2}$ in both (6) and (7) to obtain the remaining relations for both characteristics 3 and 5 . We write these as

$$
\begin{aligned}
S_{1 / 2}\left(y_{1}\right) T_{1 / 2}\left(z_{0}\right) & =T_{1 / 2}\left(z_{0}\right) S_{1 / 2}\left(y_{1}\right), \\
{\left[w_{1 / 2} T_{1}\left(z_{0}\right)\right] y_{1} } & =2 w_{1 / 2} S_{1 / 2}\left(y_{1}\right) T_{1}\left(z_{0}\right), \\
{\left[w_{1 / 2} S_{0}\left(y_{1}\right)\right] z_{0} } & =2 w_{1 / 2} T_{1 / 2}\left(z_{0}\right) S_{0}\left(y_{1}\right) .
\end{aligned}
$$

Relations (16) to (19) now hold for any characteristic prime to 2.

We may remark that Lemmas 1 and 2 of [3] now follow without change.

6. Development of the essential machinery. In this section we shall derive the machinery needed to obtain the major results of [3] in our cases. Some of the relations we shall derive are used in [3] but our cases will require a more complete development of this theory.

If $\mathfrak{A}$ is a strictly power-associative ring we have relation (1). Let $u$ be an idempotent in $\mathfrak{A}$ and let $y=u$ in (1). It then follows as in [3] that

$$
x_{1 / 2} S_{1 / 2}\left(w_{1}\right)=x_{1 / 2} T_{1 / 2}\left(w_{0}\right), \quad x_{1 / 2}^{2}=w_{1}+w_{0},
$$

and that the quantities of $\mathfrak{A}_{u}(1 / 2)$ are nilpotent if $u$ is a principal idempotent.

We now proceed to obtain other relations for later use. Consider $y_{1 / 2}$ in $\mathfrak{A}_{u}(1 / 2)$ and $g_{0}$ in $\mathfrak{H}_{u}(0)$. Define products by

$$
\begin{aligned}
& y_{1 / 2} g_{0}=b_{1 / 2}+b_{1}, \quad b_{1 / 2}=y_{1 / 2} T_{1 / 2}\left(g_{0}\right), \quad b_{1}=y_{1 / 2} T_{1}\left(g_{0}\right) ; \\
& b_{1 / 2} g_{0}=c_{1 / 2}+c_{1}, \quad c_{1 / 2}=b_{1 / 2} T_{1 / 2}\left(g_{0}\right), \quad c_{1}=b_{1 / 2} T_{1}\left(g_{0}\right) ; \\
& y_{1 / 2}^{2}=w_{1}+w_{0}, \\
& y_{1 / 2} b_{1 / 2}=z_{1}+z_{0} \text {, } \\
& y_{1 / 2} c_{1 / 2}=s_{1}+s_{0} \text {. }
\end{aligned}
$$

Then $y=z=y_{1 / 2}$, and $x=g_{0}$ in (2) yields $2 y_{1 / 2}^{2} g_{0}^{2}+4\left(y_{1 / 2} g_{0}\right)^{2}=\left(y_{1 / 2}^{2} g_{0}\right) g_{0}$ $+2\left[\left(y_{1 / 2} g_{0}\right) y_{1 / 2}\right] g_{0}+2\left[\left(y_{1 / 2} g_{0}\right) g_{0}\right] y_{1 / 2}+\left(y_{1 / 2} g_{0}^{2}\right) y_{1 / 2}$ from which we obtain three identities by considering components. We use (17) to write the component in $\mathfrak{A}_{u}(1 / 2)$ as

$$
y_{1 / 2} T_{1 / 2}\left(g_{0}\right) S_{1 / 2}\left(b_{1}\right)=y_{1 / 2} S_{1 / 2}\left(c_{1}\right) .
$$

The above substitution gives (22) only for characteristic prime to 6 . When $\mathfrak{A}$ has characteristic 3 it is necessary to make the substitution $x=g_{0}, y=z=y_{1 / 2}$, $w=u$ in (6) to obtain (22). The relation (19) is used to simplify the $\mathfrak{A}_{u}(0)$ and $\mathfrak{A}_{u}(1)$ components and we then have

$$
\begin{aligned}
2 w_{0} g_{0}^{2}+4\left(b_{1 / 2}^{2}\right)_{0}+ & 2\left[y_{1 / 2} S_{0}\left(b_{1}\right)\right] g_{0}=\left(w_{0} g_{0}\right) g_{0}+2 z_{0} g_{0}+4 s_{0}+6 y_{1 / 2} S_{0}\left(c_{1}\right), \\
4\left(b_{1 / 2}^{2}\right) u+3 b_{1}^{2} & =4\left(y_{1 / 2} c_{1 / 2}\right) u,
\end{aligned}
$$


where $\left(b_{1 / 2}^{2}\right)_{0}$ is the component of $b_{1 / 2}^{2}$ in $\mathfrak{A}_{u}(0)$.

It will be frequently useful to have identities obtained by making the substitutions $x=y=y_{1 / 2}, z=g_{0}, w=u$ and $x=y=y_{1 / 2}, z=g_{0}^{2}, w=u$. These are

$$
2 z_{0}+y_{1 / 2} S_{0}\left(b_{1}\right)=w_{0} g_{0}
$$

and

$$
4 s_{0}+4 y_{1 / 2} S_{0}\left(c_{1}\right)=w_{0} g_{0} .
$$

Finally we obtain a relation which is crucial in proving two of our most important theorems. Let $x=y_{1 / 2}, y=z=g_{0}$ in (5) to obtain $4\left[\left(y_{1 / 2} g_{0}\right) g_{0}\right] y_{1 / 2}^{2}$ $+2\left(y_{1 / 2} g_{0}^{2}\right) y_{1 / 2}^{2}+8\left[\left(y_{1 / 2} g_{0}\right) y_{1 / 2}\right]\left(y_{1 / 2} g_{0}\right)+4\left(y_{1 / 2}^{2} g_{0}\right)\left(y_{1 / 2} g_{0}\right)+2 y_{1 / 2}^{3} g_{0}^{2}=4\left\{\left[\left(y_{1 / 2} g_{0}\right)\right.\right.$ $\left.\left.\cdot y_{1 / 2}\right] y_{1 / 2}\right\} g_{0}+4\left\{\left[\left(y_{1 / 2} g_{0}\right) y_{1 / 2}\right] g_{0}\right\} y_{1 / 2}+4\left\{\left[\left(y_{1 / 2} g_{0}\right) g_{0}\right] y_{1 / 2}\right\} y_{1 / 2}+2\left[\left(y_{1 / 2} g_{0}^{2}\right) y_{1 / 2}\right] y_{1 / 2}$ $+2\left[\left(y_{1 / 2}^{2} g_{0}\right) y_{1 / 2}\right] g_{0}+2\left(y_{1 / 2}^{3} g_{0}\right) g_{0}+2\left[\left(y_{1 / 2}^{2} g_{0}\right) g_{0}\right] y_{1 / 2}$. Then compute the component of this expression in $\mathfrak{A}_{u}(1 / 2)$, apply the transformation $S_{1 / 2}\left(b_{1}\right) T_{1}\left(g_{0}\right)$ to it, use (17) to (22), (25), (26) and write the result as

$$
\begin{aligned}
b_{1}^{4}+2\left(c_{1} z_{1}\right) b_{1}+8\left[y_{1 / 2} T_{1 / 2}\left(g_{0}\right) T_{1 / 2}\left(w_{0}\right) T_{1}\left(g_{0}\right)\right] c_{1} \\
+ \\
\quad+2\left[y_{1 / 2} T_{1 / 2}\left[y_{1 / 2} S_{0}\left(b_{1}\right)\right] T_{1}\left(g_{0}\right)\right] c_{1} \\
=2\left(w_{1} c_{1}\right) c_{1}+4\left(b_{1} s_{1}\right) b_{1}+4\left[y_{1 / 2} T_{1 / 2}\left[y_{1 / 2} S_{0}\left(b_{1}\right)\right] T_{1 / 2}\left(g_{0}\right) T_{1}\left(g_{0}\right)\right] b_{1} \\
\quad+4\left[y_{1 / 2} T_{1 / 2}\left[y_{1 / 2} S_{0}\left(c_{1}\right)\right] T_{1}\left(g_{0}\right)\right] b_{1} .
\end{aligned}
$$

This completes our set of relations.

7. Decomposition relative to a set of idempotents. The decomposition of a ring relative to a set of pairwise orthogonal idempotents depends on

Lemma 1. Let $u$ and $v$ be orthogonal idempotents of a power-associative ring $\mathfrak{A}$. Then $(a u) v=(a v) u$ for every $a$ of $\mathfrak{A}$.

For proof write $\mathfrak{A}=\mathfrak{A}_{u}(1)+\mathfrak{A}_{u}(1 / 2)+\mathfrak{A}_{u}(0), \quad a=a_{1}+a_{1 / 2}+a_{0}$, and $a_{1 / 2} v$ $=b_{1}+b_{1 / 2}$. Using these relations it is seen by direct computation that $2 R_{u}^{2} R_{v}$ $=R_{u} R_{v}, 2 R_{u} R_{v} R_{u}=R_{v} R_{u}$, and $2 R_{v} R_{u}^{2}=3 R_{v} R_{u}-2 R_{u} R_{v}$ and, by the symmetry of $u$ and $v, 2 R_{v}^{2} R_{u}=2 R_{v} R_{u}, 2 R_{v} R_{u} R_{v}=R_{u} R_{v}$ and $2 R_{u} R_{v}^{2}=3 R_{u} R_{v}-2 R_{v} R_{u}$. We also have $R_{u} R_{v} R_{u} R_{v}=R_{u}^{2} R_{v}^{2}$ and, using the preceding relations, $4 R_{u}\left(R_{v} R_{u} R_{v}\right)$ $=2 R_{u}^{2} R_{v}=R_{u} R_{v}, 4 R_{u}^{2} R_{v}^{2}=2 R_{u} R_{v}^{2}=3 R_{u} R_{v}-2 R_{v} R_{u}$. Thus $R_{u} R_{v}=3 R_{u} R_{v}-2 R_{v} R_{u}$, $2 R_{v} R_{u}=2 R_{u} R_{v}$ as desired.

The proof of Lemma 1 also implies

Lemma 2. Let $u$ and $v$ be orthogonal idempotents. Then $(a u) v=(a v) u$ is contained in the intersection of $\mathfrak{A}_{u}(1 / 2)$ and $\mathfrak{A}_{v}(1 / 2)$ for every a of $\mathfrak{A}$.

The remainder of the work in [3] is now valid without change for the case when the characteristic is 5 . When the characteristic is 3 , the material in [3] from Lemma 4 to Lemma 10 holds, and there is no need for us to repeat those results here. 
8. Simple algebras. If $\mathfrak{A}$ is a commutative power-associative algebra with unity quantity $e=u+v$ where $u$ and $v$ are orthogonal idempotents, we may write $\mathfrak{A}=\mathfrak{A}_{1}+\mathfrak{A}_{12}+\mathfrak{H}_{2}$ where $\mathfrak{A}_{1}=\mathfrak{A}_{u}(1)=\mathfrak{A}_{v}(0), \mathfrak{A}_{12}=\mathfrak{A}_{u}(1 / 2)=\mathfrak{A}_{v}(1 / 2), \mathfrak{A}_{2}$ $=\mathfrak{A}_{u}(0)=\mathfrak{A}_{v}(1)$ and make corresponding changes in the subscripts of the elements of $\mathfrak{A}$ and transformations $S_{\lambda}, T_{\mu}$ as in [3]. It is known $\left(^{3}\right)$ that $\mathfrak{A}_{1}=u \mathfrak{F}$ $+\mathfrak{G}_{1}, \mathfrak{A}_{2}=v \mathfrak{F}+\mathfrak{B H}_{2}$ where $\mathfrak{B}=\mathfrak{G}_{1}+\mathfrak{G}_{2}$ is a nilalgebra. Also, $x y=a e+g$ for every $x$ and $y$ of $\mathfrak{A}_{12}$ where $a$ is in $\mathfrak{F}$ and $g$ is in (\$).

We are now in a position to prove Theorem 3 of [3] for algebras of characteristic 3 . The result may then be stated as

THEOREM 4. Every simple commutative strictly power-associative algebra of characteristic not two is either an algebra of degree one or two with a unity quantity or is a Jordan algebra.

Since the decomposition relative to an idempotent and relations (16) to (19) have been shown for algebras of characteristic 5, the proof of [3] is now valid for these algebras. When the characteristic is 3 it is necessary only to provide a proof for the following important lemma.

Lemma 3. Let $y_{12} S_{2}\left(x_{1}\right)$ be in $\mathfrak{S}_{2}$ for every $x_{1}$ of $\mathfrak{A}_{1}$ and $y_{12}$ of $\mathfrak{A}_{12}$. Then $y_{12} T_{1}\left(x_{2}\right)$ is in $\mathfrak{G}_{1}$ for every $x_{2}$ of $\mathfrak{A}_{2}$ and $y_{12}$ of $\mathfrak{A}_{12}$.

Suppose the lemma were not true. Since $y_{12} T_{1}(v)=0$, we may then assume that there exist a $y_{12}$ and a $g_{2}$ such that $y_{12} T_{1}\left(g_{2}\right)$ is not in $\left(\xi_{1}\right.$. As in [3] we may take $y_{12} T_{1}\left(g_{2}\right)=u$. Then with $b_{1}=u$ and with appropriate changes in subscripts to fit the notations of this section, relation (27) becomes

$$
\begin{aligned}
u+2 c_{1} z_{1}+2\left[y_{1 / 2} T_{12}\left(g_{2}\right)\right. & \left.T_{12}\left(w_{2}\right) T_{1}\left(g_{2}\right)\right] c_{1} \\
= & 2\left(w_{1} c_{1}\right) c_{1}+s_{1}+y_{12} T_{1 / 2}\left[y_{12} S_{2}\left(c_{1}\right)\right] T_{1}\left(g_{2}\right) .
\end{aligned}
$$

We shall arrive at a contradiction by showing that $u$ is in (s).

From (26) and the hypothesis of the lemma, $s_{2}$ is in $\mathbb{G}_{2}$ and it follows that $s_{1}$ is in $\mathfrak{S}_{1}$. Using $b_{1}=u$ in (22) we obtain

$$
y_{12} T_{1 / 2}\left(g_{2}\right)=2 y_{12} S_{1 / 2}\left(c_{1}\right) \text {. }
$$

This implies $c_{1}$ is in $\mathfrak{S}_{1}$. For if $c_{1}=\gamma u+c_{1}^{\prime}$ with $c_{1}^{\prime}$ in $\mathbb{G}_{1}$, then $(1 / 2) \gamma y_{12}$ $=y_{12}\left[(1 / 2) T_{1 / 2}\left(g_{2}\right)-S_{1 / 2}\left(c_{1}^{\prime}\right)\right]$. Since $(1 / 2) T_{1 / 2}\left(g_{2}\right)-S_{1 / 2}\left(c_{1}^{\prime}\right)$ is a nilpotent transformation, we must have $\gamma=0$. The component in $\mathfrak{A}_{1}$ of the relation obtained by letting $x=b_{12}, y=y_{12}, z=c_{1}, w=v$ in (3) gives

$$
y_{12} T_{1 / 2}\left[y_{12} S_{2}\left(c_{1}\right)\right] T_{1}\left(g_{2}\right)=2 b_{12}^{2} u+y_{12} T_{1 / 2}\left(g_{2}\right) T_{1}\left[y_{12} S_{2}\left(c_{1}\right)\right]+2 s_{1}+2 z_{1} c_{1} .
$$

The term $b_{12}^{2} u$ is in $G_{1}$ by (23) and $y_{12} T_{1 / 2}\left(g_{2}\right) T_{1}\left[y_{12} S_{2}\left(c_{1}\right)\right]=2 y_{12} S_{1 / 2}\left(c_{1}\right)$ - $T_{1}\left[y_{12} S_{2}\left(c_{1}\right)\right]=\left\{y_{12} T_{1}\left[y_{12} S_{2}\left(c_{1}\right)\right]\right\} c_{1}$ is in $G_{1}$. Thus $y_{12} T_{1 / 2}\left[y_{12} S_{2}\left(c_{1}\right)\right] T_{1}\left(g_{2}\right)$ is in $\mathfrak{G}_{1}$ and $u$ is in $\mathfrak{G}_{1}$. This completes the proof.

(3) [3, Lemma 10]. 
9. The general structure theory. The general structure theory for powerassociative algebras depends on Theorem 7 of [3] and its consequences. After we prove it for algebras of characteristic 3 we may state the complete result as

THEOREM 5. Let e be a principal idempotent of a commutative strictly powerassociative algebra $\mathfrak{A}$ of characteristic not two. Then $\mathfrak{A}_{e}(1 / 2)+\mathfrak{H}_{e}(0)$ is contained in the radical of $\mathfrak{A}$.

When the characteristic is 3 all of the proof given in [3] is valid except that part where $\mathfrak{A}$ is assumed to be semisimple and $\mathfrak{D} \neq \mathfrak{A}$ is a nonzero ideal of $\mathfrak{A}$. It is then only necessary to prove $\mathfrak{D}$ is semisimple and to show this we need to demonstrate that $\mathfrak{D}_{e}(1 / 2) \mathfrak{A}_{e}(0) \subseteq \mathfrak{M}$ where $\mathfrak{M}$ is the radical of $\mathfrak{D}$ and $e$ is a principal idempotent of $\mathfrak{D}$. And then it is sufficient to prove $b_{1}=y_{1 / 2} T_{1}\left(g_{0}\right)$ is in $\mathfrak{M}$ for $y_{1 / 2}$ in $\mathfrak{D}_{e}(1 / 2)$ and $g_{0}$ in $\mathfrak{A}_{e}(0)$. We shall show that $b_{1}^{4}$ is in $\mathfrak{M}$ since then it will follow as in [3] that $b_{1}$ is in $\mathfrak{M}$.

With products defined as in (21), except that the subscripts are relative to $e$, we have (27). Next let $x=y=y_{1 / 2}, z=g_{0}, w=b$, in (3) to obtain the two relations

$$
\begin{gathered}
y_{1 / 2} T_{1 / 2}\left(g_{0}\right) T_{1 / 2}\left[y_{1 / 2} S_{0}\left(b_{1}\right)\right]=y_{1 / 2} T_{1 / 2}\left[y_{1 / 2} S_{0}\left(b_{1}\right)\right] T_{1 / 2}\left(g_{0}\right), \\
y_{1 / 2} T_{1 / 2}\left(g_{0}\right) T_{1}\left[y_{1 / 2} S_{0}\left(b_{1}\right)\right]+\left[b_{1 / 2}\left(y_{1 / 2} S_{1 / 2}\left(b_{1}\right)\right)\right] e \\
\quad=2\left[y_{1 / 2}\left(b_{1 / 2} S_{1 / 2}\left(b_{1}\right)\right)\right] e+y_{1 / 2} T_{1 / 2}\left[y_{1 / 2} S_{0}\left(b_{1}\right)\right] T_{1}\left(g_{0}\right)+\left(y_{1 / 2} b_{1 / 2}\right) b_{1} .
\end{gathered}
$$

The element $y_{1 / 2}$ is in $\mathfrak{D}_{1 / 2} \subseteq \mathfrak{M}$ and $c_{1}$ is in $\mathfrak{D}_{1}$ so $y_{1 / 2} S_{0}\left(c_{1}\right)$ is in $\mathfrak{M}$. Since $\mathfrak{D}_{1 / 2} \mathfrak{D}_{1 / 2} \subseteq \mathfrak{M}$ the last relation implies $y_{1 / 2} T_{1 / 2}\left[y_{1 / 2} S_{0}\left(b_{1}\right)\right] T_{1}\left(g_{0}\right)$ is in $\mathfrak{M}$. Similarly a relation obtained by the substitution $x=y=y_{1 / 2}, z=g_{0}, w=c$, in (3) implies $y_{1 / 2} T_{1 / 2}\left[y_{1 / 2} S_{0}\left(c_{1}\right)\right] T_{1}\left(g_{0}\right)$ is in $\mathfrak{M}$. Also (30), (19), (18), and (22) give

$$
\left[y_{1 / 2} T_{1 / 2}\left[y_{1 / 2} S_{0}\left(b_{1}\right)\right] T_{1 / 2}\left(g_{0}\right) T_{1}\left(g_{0}\right)\right] b_{1}=\left[y_{1 / 2} T_{1 / 2}\left[y_{1 / 2} S_{0}\left(b_{1}\right)\right] T_{1}\left(g_{0}\right)\right] c_{1} \text { in } \mathfrak{M} \text {. }
$$

Finally we need the relation

$$
2 y_{1 / 2} T_{1 / 2}\left(g_{0}\right) T_{1 / 2}\left(w_{0}\right)=y_{1 / 2} T_{1 / 2}\left[y_{1 / 2} S_{0}\left(b_{1}\right)\right]+2 y_{1 / 2} S_{1 / 2}\left(z_{1}\right)
$$

which is obtained by setting $x=y=y_{1 / 2}, z=b_{1 / 2}, w=e$ in (3). After observing that $z_{1}$, $w_{1}$, and $s_{1}$ are in $\mathfrak{D}_{1 / 2} \mathfrak{D}_{1 / 2} \subseteq \mathfrak{M}$, it follows from (27) that $b_{1}^{4}$ is in $\mathfrak{M}$ as desired.

The associativity of fifth powers was used twice in the proof of Theorem 5; namely, in obtaining (22) and (27). To show the necessity of using fifth power associativity when the characteristic is 3 we give an example. Let $\mathfrak{A}$ be a commutative algebra over a field $\mathfrak{F}$ whose characteristic is 3 with a basis of three elements $e, y, g$. Define multiplication in $\mathfrak{A}$ by $e^{2}=e, e y=2 y, y g=e$, and $e g=y^{2}=g^{2}=0$. Then $\mathfrak{A}_{e}(1)=e \mathfrak{F}, \mathfrak{A}_{e}(1 / 2)=y \mathfrak{F}$, and $\mathfrak{A}_{e}(0)=g \mathfrak{F}$. Direct computation shows that fourth powers are associative but the example contradicts the conclusion of the theorem since $\mathfrak{A}_{e}(1 / 2) \mathfrak{H}_{e}(0)=\mathfrak{A}_{e}(1)$. Of course fifth powers cannot be associative. 
10. Algebras of degree 2 and characteristic 0 . We go back to algebras of degree 2 and shall again use the notations of $\$ 8$. Since simple commutative power-associative algebras of degree greater than two are classical Jordan algebras, it seemed natural to conjecture that simple algebras of degree two are also Jordan algebras. It has been shown by the construction of examples $[4 ; 5]$ that this conjecture is false for algebras of characteristic $p$. However, it is known [4] that stable simple algebras of degree two and characteristic 0 are Jordan algebras. No decisive result has been obtained for algebras of characteristic 0 and not necessarily stable, but, in attempting to prove that they are Jordan algebras, some interesting results have been found. If $\mathfrak{M}=\mathfrak{H}+\mathfrak{A}_{12} S_{1 / 2}\left(\mathfrak{S}_{1}\right)+\mathfrak{A}_{12} T_{1 / 2}\left(\mathfrak{S}_{2}\right)$ can be shown to be an ideal of a simple algebra $\mathfrak{A}$, it would follow that $\mathfrak{A}$ is a classical Jordan algebra. We are not able to show $\mathfrak{M}$ is an ideal but in the attempt to do so have obtained a stronger form of Lemma 3 for algebras of characteristic zero. This result is false when the characteristic is $p[5]$.

We now prove the preliminary

LEMMA 4. Let $\mathfrak{A}$ be a commutative strictly power-associative algebra whose characteristic is prime to 6. If $y_{12} T_{1}\left(g_{2}\right)=u$ for some $y_{12}$ in $\mathfrak{A}_{12}$ and some $g_{2}$ in $\mathfrak{P}_{2}$, then $y_{12} S_{2}\left[y_{12} T_{1 / 2}\left(g_{2}\right) T_{1}\left(g_{2}\right)\right]$ is either 0 or $(-1 / 2) v$.

By hypothesis we have (21) and (29). These and relations (18) and (19) imply

$$
\begin{aligned}
& y_{12} c_{1}=(1 / 2) b_{12}+b_{2}, \quad b_{12} c_{1}=(1 / 2) c_{12}+(1 / 2 b)_{2} g_{2}, \\
& y_{12} c_{1}^{2}=(1 / 2) c_{12}+b_{2} g_{2} .
\end{aligned}
$$

Let $x=y=y_{12}, z=c_{1}$ in (3). Then taking $w=c_{1}$ and $w=g_{2}$ in turn we obtain

$$
2 b_{12}^{2} v=2 s_{2}-6 b_{2}^{2}=s_{2}+(1 / 2) z_{2} g_{2}+2 b_{2} .
$$

Eliminating $b_{12}^{2} v$ between (23) and (33) yields $2 s_{2}+2 b_{2}+z_{2} g_{2}+\left(w_{2} g_{2}\right) g_{2}-2 w_{2} g_{2}^{2}$ $=0$ which, when combined with (25) and (26), gives $\left(w_{2} g_{2}\right) g_{2}=w_{2} g_{2}^{2}$. It follows from this fact and (25), (26), (33) that $6 b_{2}^{2}+3 b_{2}=0$ and $-2 b_{2}$ is either 0 or an idempotent of $\mathfrak{A}_{2}$. But $v$ is an absolutely primitive idempotent of $\mathfrak{A}_{2}$ so $-2 b_{2}=0$ or $v$.

It is possible to prove Lemma 4 for an algebra whose characteristic is 3 , but, since we shall not use the result, we shall not prove it.

The hypothesis of Lemma 3 was necessary because we needed to have $b_{2}$ in $\mathfrak{G}_{2}$. Thus when $b_{2}=0$ the hypothesis of Lemma 3 is satisfied and so we may assume $b_{2}=-(1 / 2) v$ in the proof of the following stronger result.

TheOREm 6. Let $\mathfrak{A}$ be a power-associative algebra of degree 2 and characteristic 0 . Then $y_{12} T_{1}\left(x_{2}\right)$ is in $\mathfrak{S}_{1}$ for every $y_{12}$ of $\mathfrak{A}_{12}$ and $x_{2}$ of $\mathfrak{A}_{2}$. By symmetry $y_{12} S_{2}\left(x_{1}\right)$ is in $\mathfrak{S}_{2}$ for every $y_{12}$ of $\mathfrak{A}_{12}$ and $x_{1}$ of $\mathfrak{A}_{1}$. 
Suppose the theorem were not true. Then there exist a $y_{12}$ and a $g_{2}$ such that $y_{12} T_{1}\left(g_{2}\right)=u$, Lemma 4 applies, and, as we have observed, we may take $b_{2}=y_{12} S_{2}\left(c_{1}\right)=-(1 / 2) v$.

The element $g_{2}$ is nilpotent so there exists a least integer $k$ such that $g^{k}=0$. Consequently, by (17), (29), and (19), $y_{12} T_{1 / 2}\left(g_{2}^{k}\right)=0=2^{k-1} y_{12}\left[T_{1 / 2}\left(g_{2}\right)\right]^{k}$ $\left.=y_{12} T_{1 / 2}\left(g_{2}\right)\right]^{k} T_{1}\left(g_{2}\right)=2^{k} y_{12}\left[S_{1 / 2}\left(c_{1}\right)\right]^{k} T_{1}\left(g_{2}\right)=c_{1}^{k}$. Furthermore, $c_{1}^{k}=0$ implies

$$
\begin{aligned}
y_{12} S_{2}\left(c_{1}^{k}\right)=0 & =k \cdot 2^{k-1} y_{12}\left[S_{1 / 2}\left(c_{1}\right)\right]^{k-1} S_{2}\left(c_{1}\right) \\
& =k y_{12}\left[T_{1 / 2}\left(g_{2}\right)\right]^{k-1} S_{2}\left(c_{1}\right)=-k 2^{-k} g_{2}^{k-1}
\end{aligned}
$$

by (16), (29), and (19). The algebra has characteristic 0 so $g_{2}^{k-1}=0$ contradicting the fact that $k$ was chosen to be the index of nilpotency of $g_{2}$.

It is evident that the proof of Theorem 6 breaks down for characteristic $p$ since $g_{2}^{k}=0$ implies $k g_{2}^{k-1}$ and it may be that $k$ is a multiple of $p$. In other words, it is not possible to find $y_{12}$ and $g_{2}$ such that $y_{12} T_{1}\left(g_{2}\right)=u$ unless the index of nilpotency of $g_{2}$ is a multiple of the characteristic. These facts were used in constructing simple power-associative commutative algebras of degree 2 and characteristic $p$ which are not Jordan algebras [5].

\section{BIBLIOGRAPHY}

1. A. A. Albert, On the power-associativity of rings, Summa Braziliensis Mathematicae vol. 2 (1948) pp. 21-33.

2. - Power-associative rings, Trans. Amer. Math. Soc. vol. 64 (1948) pp. 552-593.

3. - A theory of power-associative commutative algebras, Trans. Amer. Math. Soc. vol. 69 (1950) pp. 503-527.

4. - On commutative power-associative algebras of degree two, Trans. Amer. Math. Soc. vol. 74 (1953) pp. 323-343.

5. L. A. Kokoris, Power-associative commutative algebras of degree two, Proc. Nat. Acad. Sci. U.S.A. vol. 38 (1952) pp. 534-537.

UNIVERSITY OF WASHINGTON, Seattle, Wash. 